\title{
LA PEDAGOGÍA HISTÓRICO-CRÍTICA: UNA ALTERNATIVA DE CAMBIO EN LAS INSTITUCIONES EDUCATIVAS DE TACNA
}

Responsable : Mgr. Adriana Luque Ticona
Miembro: Antrop. Isaias Rey Pérez Alférez

\section{RESUMEN}

La educación es un proceso en el cual diferentes sujetos entran en relación y en cuyo proceso de relación se generan ciertos cambios al interior de cada uno de ellos. Es un hecho aceptar que la intensidad de los cambios de la vida social está asociada a la modalidad de relación por la que se opte en el sistema educativo. Este supuesto, en torno al impacto social del hecho educativo, está presente en casi todas las escuelas de pensamiento, las que de una u otra forma tienden a dar una respuesta a este problema educativo. Una de esas tendencias considera que la educación debe darse en un contexto, con los elementos culturales de ese contexto, de manera que el aprendizaje adquiera mayor relación y significación. La pedagogía histórico-crítica se inscribe dentro de este pensamiento. Según el concepto de los escolares, el aprendizaje tendria mejores resultados con la utilización de las experiencias, lenguaje, hechos y fenómenos del entorno. Así, los contenidos tendrían mayor relación y, por tanto, los educandos lograrian desarrollar mejor sus capacidades.

\section{ABSTRACT}

The education is a process which different subjects enter relation and whose process of relation certain changes to the interior of each one of them are generated. It is a make accept that the intensity of the changes of the social life is associate to the relation modality by which it is chosen in the educative system. This assumption, around the social impact of the educative fact, is present in almost all the schools of thought, those that of one or another form tend to give an answer to this educative problem. One of those tendencies considers that the education must occur in a context with the cultural elements of that context, so that the learning acquires greater relation and meaning. Pedagogia historicalcritic registers within this thought. According to the concept of the students, the learning would have better results with

\section{LA EDUCACIÓN NUEVA O EDUCACIÓN ACTIVA}

En las últimas décadas del siglo pasado se ha producido una verdadera revolución en el sistema educativo en los distintos paises, ante dos fuerzas sociales poderosas, intimamente relacionadas entre sí, que son, la urbanización y la industrialización, que motivaron sistemas educativos actualizados. La educación se encuentra considerablemente implicada en estos cambios. Por lo tanto, ella es una fuerza social que actúa con los cambios sociales $\mathrm{y}$, al mismo tiempo, aplicada a cambiar la misma sociedad donde actúa.

El surgimiento de la pedagogía contemporánea puede considerarse como una reacción contra la concepción que imperó hasta el siglo XIX. Al comenzar el siglo XX se producen movimientos pedagógicos, principalmente los de Dewey, quien pretendió darle a la educación y a la pedagogía un sentido activo y vital; el de Paul Natorp, quien estableció que el hombre sólo se hace hombre mediante la sociedad humana; aspira a renovar la concepción social de la educación y de la pedagogia; el de Ellen Key, defiende la vida infantil. Para Key, la educación consiste en dejar que la naturaleza actúe lenta y tranquilamente sobre el alma del niño, y en tratar sólo que las circunstancias del ambiente apoyen el trabajo de la naturaleza.

En todo caso, se puede concluir que durante el siglo XX surgen varias corrientes pedagógicas; algunas tuvieron su origen en siglos anteriores. entre estas pueden citarse: la pedagogía psicológica experimental, la pedagogía activa, la pedagogía individual, la pedagogía social, pedagogia filosófica, etc.

\section{Educación y Sociedad:}

Ciertamente, no puede negarse que de toda actividad educativa se sigue una consecuencia social. No es posible teorizar en educación dejando de lado el estudio del problema social, así como el problema de las relaciones de poder y sus implicaciones en la educación, o sin responder al problema antropológico en torno a la naturaleza humana y sus diversas implicaciones en el hecho educativo

Como dice Noé Morales Rodríguez, quizás una de las preguntas fundamentales que debe hacerse el teórico de la educación o el educador mismo es : ¿cuál es el modelo de sociedad que estimula con su modelo educativo? O más aún: ¿de qué modo la modalidad educativa que desarrolla afecta o no las condiciones de vida social de los educandos o del sistema social en que ellos están inmersos? En este sentido, es posible que surjan dos tipos de categorías de respuesta:

Por un lado, aquellas que hablan de modelos de búsqueda de superación de la desigualdad entre los seres humanos, y, por ende, de integración entre estos, en donde la condición de marginalidad es algo que debe ser superado, ya que en si misma 
encierra la negación de lo que se estimaria como justo desde una perspectiva político-social. La misión de la educación seria, entonces, la de promover la superación de la condición de desigualdad, o como afirma Saviani "La educación emerge alli como un instrumento de corrección de estas distorsiones". En este sentido, la educación es la fuerza homogeneizadora que refuerza los lazos sociales, promueve la cohesión y garantiza la integración social.

Por otro lado, habria que ubicar aquellas teorías que conciben la sociedad como un todo organizado por clases o estamentos, en donde la posesión de los bienes de producción y el acceso al poder es una prerrogativa de un grupo reducido, en detrimento de una gran mayoría que no goza de tales beneficios, dada su condición de marginalidad. Normalmente en esta opción, quien ostenta el poder económico ejerce el poder político y, al mismo tiempo, posee el conocimiento o ejerce control sobre él. De este modo, quien ostenta el poder legitima su condición mediante determinados instrumentos. Aquí, el papel que le corresponde a la educación es el de legitimar la condición social de la marginalidad como condición inherente a la estructura social, y como consecuencia "legítima" de la libertad de oportunidades. La educación reproduce en su interior lo que sucede en la sociedad y opera como un mecanismo de ratificación del estado de las cosas.

De lo anterior, fácilmente se infiere que la educación es una experiencia eminentemente política. No está divorciada la escuela de la sociedad y tampoco lo está de los grupos o clases que ostentan el poder. El papel oficial que debe cumplir la educación está determinado por el ejercicio del poder. Es por ello que a todo modelo social corresponde una modalidad de organización educativa, ya sea ésta conservadora o revolucionaria, ya sea ésta de izquierda o de derecha, ya sea ésta dictatorial u oligárquica; en cualquier caso, el ejercicio del poder supone, como contraparte, una modalidad educativa que le corresponde y que lo ratifica.

En tal sentido, para efectos de análisis se puede considerar dos macrogrupos, el primero que busca la superación de la marginalidad y el segundo que justifica su existencia, Saviani denomina con los apelativos de "Teorías no-críticas" a aquellas escuelas que tienen por objetivo la legitimación del poder, y "Teorias críticas" a las que tienen por objetivo la superación de la desigualdad. No obstante lo anterior, existe un tercer grupo de teorias que son las "Crítico-reproductivistas", las cuales manifiestan un discurso de cambio, pero que, de algún modo, no son capaces de llegar a generar las condiciones necesarias para llevarlo a cabo. Si bien es cierto, estas teorías hablan de cambio, su planteamiento es meramente retórico, por lo que ideológicamente está más cerca de las teorias nocríticas.

\section{Teoria de la Escuela como Aparato Ideológico del Estado.}

Para Althusser, el Estado se impone a base de dos conjuntos de aparatos (sistemas de acción). Por un lado están los "aparatos represivos del Estado", constituido por los cuerpos de seguridad, los tribunales, las cárceles, el propio gobierno, entre otros. Por otro lado, están los "aparatos ideológicos del Estado", constituido por la familia, la religión, la escuela, el sistema politico, los sindicatos, los medios de información, la cultura y el aparato juridico.

Los aparatos represivos actúan en forma directa y suelen imponerse por la vía de la fuerza. Los aparatos ideológicos del Estado utilizan una vía sutil y a veces subliminal, es la vía de la violencia simbólica; por ello se les llama ideológicos. Su objetivo es convencer y procuran hacerlo de forma que no se perciba que se está promoviendo una labor de convicción. El sistema educativo se ubica dentro del conjunto de aparatos ideológicos; la escuela, en lugar de promover la igualdad social, se constituye en instrumento para garantizar y perpetuar los intereses de quien ostenta el poder.

De todos los aparatos ideológicos del Estado, la escuela es la que se puede concebir como el instrumento más desarrollado y de mayor penetración. Todos los niños y niñas, de todo el conglomerado social, deben pasar por el sistema educativo. Los conocimientos y valores que se les infunde, durante los años de educación obligatoria van a desembocar en una mentalidad y por ende en un resultado ideológico.

El problema para lograr la ruptura de este modelo, radica en el hecho de que la condición de marginalidad está interiorizado en cada uno de los sujetos alienados, los cuales aceptan como normal su situación dada la influencia que en ese sentido han tenido los mecanismos ideológicos. No debe olvidarse que, en buena medida, la mentalidad es un juego entre la experiencia individual y la vivencia social organizada. Esta última, al estar en manos de quien condiciona lo que debo pensar, obstaculiza el desarrollo de la lucha para romper y modificar la condición de marginalidad.

Uno de los problemas de este enfoque es que tiende a ver los procesos sin sujeto, sólo los ve como estructuras en el ámbito superior, especie de macroconcepciones metafísicas al servicio de determinados intereses, pero sin llegar a señalar a los sujetos concretos que las manipulan.

\section{Enfoque Teórico - Crítico.}

Según N. Morales, si miramos el desarrollo del hombre, ya sea en su historia personal o en la historia de la humanidad, será evidente para nosotros que solemos pasar de una situación de reto a otra. Las etapas precedentes son parte del tinglado de las nuevas situaciones y los nuevos 
retos que asumiremos, existen en la medida en que son reales las vivencias actuales. Las respuestas que hoy obtenemos encierran en si mismas las interrogantes de la fase siguiente. Este es un proceso dialéctico que opera de forma tal que lo que en determinado momento se afirma como válido, entrará en crisis y generará su propia negación; como resultado de este proceso de confrontación se planteará una nueva respuesta, que de algún modo contiene fases de la tesis como de la antítesis. Lo interesante es que esta sintesis es, de hecho, en si misma, una nueva tesis que tendrá su negación y asi progresivamente. En el caso de lo fortuito, lo casual - lo accidental, estos serán asumidos por la conciencia a partir de las características que tiene en su momento evolutivo.

El enfoque crítico es en sí mismo dialéctico y en ello radica su racionalidad. La progresividad de esta racionalidad dialéctica nace del hecho de que no hay respuestas finales y de que tampoco hay satisfacción plena de lo que se descubre. Lo que descubrimos de la realidad hoy, deberá modificarse en la medida de que por un lado, el sujeto del proceso cognitivo está cambiando $y$, por ende, cambia su perspectiva de análisis y, por otro lado, la realidad a la que se refiere también es cambiante, con lo cual el sujeto cognoscente también deberá actualizar sus conocimientos. Ambos, sujeto y objeto de conocimiento son procesos en movimiento y en interacción dialéctica permanente.

El marco en el que se desarrolla este proceso de crecimiento dialéctico, es el marco social. Por ello, la educación es un acto político y no un fenómeno estrictamente pedagógico. La neutralidad es sólo un supuesto, nunca una realidad. Para que el ser humano desarrolle procesos reales de conocimiento, deberá asumir un proceso en condiciones de libertad, ésta es la búsqueda de satisfacer las necesidades, pero éstas nunca estarán totalmente satisfechas; entonces, la libertad será siempre un proyecto de búsqueda. La marginalidad, deberá superarse en la medida en que se asuma el proyecto de la libertad. La lucha contra la discriminación supone generar, junto con los marginados mismos, el descubrir las condiciones de su propia situación, para determinar en ese proceso que debe descubrirse por la propia gestión de la escuela con la comunidad; la búsqueda de mejores calidades de vida. El papel de la teoría crítica, es la de dar al proceso educativo las condiciones requeridas para no estar sometida al juego de los intereses de la clase dominante.

No puede pensarse en una opción nacional de cambio en donde, éste sea, propuesto por las figuras gubernamentales y en forma similar o idéntica para todos. Las opciones de transformación deben emerger de la propia situacionalidad, con una dimensión curricular adecuada a las condiciones de necesidad de tal coyuntura. Deben responder critica y activamente a las condiciones reales de existencia y con la clara convicción de que la escuela es una de las tantas fuerzas dinámicas que participan en la búsqueda de superación del conflicto, de la injusticia, en procura de la adecuada respuesta de generar procesos en dignidad y en el rescate de procesos de optimización permanente.

Una de las posiciones de mayor impacto desde la pedagogía crítica es la desarrollada por Paulo Freire. La educación como una opción para que el marginado diga su palabra, la ruptura con la educación tradicional en procura de una educación dialógica, es quizás una de las alternativas de ruptura que más ha impactado la educación en América Latina. La praxis pedagógica entendida como educación acción, en donde se rompe el verbalismo del magistrocentrismo y el activismo de la escuela nueva. La educación acción es una pedagogía situacional a partir de las situaciones de contexto del educando. El currículo es situacional y con ello se sientan las bases para una lectura de la cultura mundial desde la propia cultura del educando. La impronta de las teorias pedagógicas de Freire se observa no solo en las diferentes manifestaciones de la pedagogia participativa, también se observan sus implicaciones en la teología de la liberación y en cierto sentido también en los Documentos de la Reunión de Obispos en Medellin.

\section{LAPEDAGOGÍAHISTÓRICO-CRÍTICA}

La Pedagogía Histórico-Crítica (PHC) es resultado de la acumulación de diversos aportes de quienes han buscado construir una educación liberadora dentro o fuera de la escuela, para el sujeto individual y colectivo; también recoge el aporte de quienes asumen críticamente el desarrollo humano y la práctica social, presentando no sólo una alternativa para los marginados y excluidos de la sociedad, sino también como andamiaje teóricoinstrumental de una manera de encarar cómo la práctica educativa contribuye al avance de las sociedades, inclusive de aquellas que se denominan desarrolladas. Una de las bases sustanciales es el pensamiento de Freire.

La Pedagogía Liberadora nace a mediados del siglo XX a propuesta del brasileño Paulo Freire (1921-1997). Parte del estudio crítico de la sociedad, lo que le da un carácter político que le niega la posibilidad de institucionalizarse en una sociedad capitalista. Esta corriente destaca con mayor énfasis el proceso de aprendizaje grupal que la calidad de los contendidos de la enseñanza.

Su propuesta de transformación de la educación, más acentuadas de perspectivas, puede ser captada en el transcurso de sus escritos, desde la publicación de su libro Pedagogía del oprimido, en 1970. El educador continuaba fiel a los temas centrales en sus reflexiones, tales como las caracteristicas de la conciencia, la critica a la educación "domesticadora", la discusión acerca de la naturaleza del hombre y del proceso de su humanización, las piedras angulares de una pedagogia libertadora, el papel central del diálogo en la educación, la participación, etc. 
Plantea duras criticas a los sislemas educativos de entonces y a las pedagogias no criticas. Llama "concepción bancaria" a la pedagogia utilizada en forma oficial, durante el siglo pasado y anteriores, ya que los conocimientos son depositados sobre los educandos que los reciben pasivamente, de ahi el término "concepción bancaria". Las relaciones que se forman en este tipo de educación son de naturaleza narrativa, están compuestas por un sujeto activo (el que narra) y uno pasivo (el educando). Los educadores son considerados "sabios" y los educandos "ignorantes".

Los contenidos de este tipo de educación son "retazos" de la realidad, están desvinculados entre si y no poseen fuerza transformadora. En estas disertaciones, la palabra se vacia de la dimensión que debe poseer, se transforma en una palabra hueca, en verbalismo alienado y alienante, por esto la característica de la educación libertadora es la "sonoridad" de la palabra y no la fuerza transformadora. En vez de comunicarse, el educador hace comunicados y depósitos de los educandos, meras incidencias, reciben pacientemente, memorizan y repiten. Tal es la concepción bancaria de la educación que el único margen de acción que ofrece a los educandos es el de recibir los depósitos, guardarlos y archivarlos, margen que les permite ser coleccionistas 0 fichadores de cosas que archivan.

Lo que pretenden los opresores "es transformar la mentalidad de los oprimidos y no la situación que los oprime". A fin de lograr una mejor adaptación a la situación que, a la vez, permite una mejor forma de dominación.

La educación bancaria tiene por fin deshumanizar al hombre, volverlo un autómata. Su acción debe estar empapada de una profunda creencia en los hombres. Creencia en su poder creador. Todo esto exige que sea, en sus relaciones con los educandos, un compañero de estos.

La educación bancaria, en cuya práctica se da la inconciliación educador-educando, rechaza este compañerismo. Esto es lógico, puesto que en el momento en que el educador bancario viviera la superación de la contradicción ya no seria "bancario", ya no se efectuarian "depósitos". Saber con los educandos en tanto estos supieran con él, sería su tarea. Ya no estaría al servicio de la deshumanización, al servicio de la opresión sino al servicio de la liberación.

\section{Concepto de Educación.}

Enseñar no puede ser un simple proceso de transferencia de conocimientos del educador al aprendiz o educando. Para Freire, la concepción de educación es la siguiente: "La educación es la praxis, reflexión y acción del hombre sobre el mundo para transformarlo". Es un acto de comunicación, un acto liberador, es una educación problematizadora, liberadora. La educación es una actividad donde profesores y alumnos, mediatizados por la realidad que aprenden, extraen contenidos de aprendizaje.

La que no le teme; sino que busca transformarla; por solidaridad, por espiritu "La educación es un acto de amor, de coraje; es una práctica de la libertad dirigida hacia la realidad a lo fraternal". Paulo Freire.

El fin principal de la educación problematizadora, es el de lograr que emerjan las conciencias de los hombres y que logren una comprensión e inserción critica en el mundo. Apunta al cambio radical, criticando la homogeneización de los individuos. El antagonismo entre las dos concepciones, la bancaria que sirve a la dominación y la problematizadora que sirve a la liberación se corporifica precisamente ahi. Mientras la primera, necesariamente, mantiene la contradicción educador- educando, la segunda realiza la superación.

\section{LINEAMIENTOS DE LA PEDAGOGÍA HISTÓRICO-CRITICA}

La Pedagogia Histórico-crítica se basa, según Libaneo, "en la línea de las sugerencias de la teoría marxista que no se satisfacen con la teoria criticoreprodutivistas y postulan una teoría critica de la educación que capte críticamente la escuela como instrumento coadyuvante en el proyecto de transformación social". Es una tentativa de superar, tantos los límites de las educaciones críticas como también el del crítico-reprodutivistas, teorías en el empeño de analizar y entender el problema educativo a partir del desarrollo histórico-objetivo. Tiene, por lo tanto, su concepción presupuesto en el materialismo histórico. Según Saviani, la educación histórica "procura retener el carácter crítico de articulación con el condicionamiento social que el reproductivista posee, ligado, sin embargo a la dimensión histórica que el reproductivismo pierde de vista".

La expresión de Pedagogía Histórico-crítica, según Saviani, sirve para entender, traducir el pasaje de la visión mecanicista ahistórica por una visión dialéctica crítica, es decir, una visión históricocrítico de la educación. El sentido básico de la Pedagogia Histórico-crítica, no es la articulación de una propuesta pedagógica que tiene el compromiso apenas de mantener la sociedad, sino de transformarla a partir de la comprensión de los condicionantes sociales y de la visión que la sociedad ejerce.

\section{EL MÉTODO DE LAPHC EN SAVIANI}

Por otro lado, el método propuesto por Saviani se sitúa más allá de los enfoques nuevos y tradicionales y, de acuerdo con ello, escribe: "deriva de una concepción que articula la educación y la sociedad, y parte de la comprobación de que la sociedad en que vivimos está dividida en clases con intereses opuestos". Saviani prefiere hablar de los 
momentos que caracterizan ese enfoque, siendo que esos momentos se deben articular en un movimiento único, cuya duración debe variar según las situaciones especificas que implican la práctica pedagógica. El primer momento o el punto de partida de la educación, no es sino la práctica social que es común a profesores y estudiantes, aunque el punto de vista pedagógico de los profesores y estudiantes pueda presentar niveles diferentes del conocimiento y la experiencia de esa práctica social. El segundo momento, está en la problematización y tiene como el objetivo identificar qué problemas serán resueltos dentro de la práctica social y qué conocimientos son necesarios para resolver esos problemas. El tercer momento es el de la instrumentalización, o sea la apropiación de los instrumentos necesarios, prácticos y teóricos para la solución de los problemas identificados, eso depende de la transmisión del conocimiento del profesor para que esa apropiación se reproduzca socialmente y sea preservada históricamente. El cuarto momento es la catarsis, que es la efectiva incorporación de los instrumentos culturales y la forma elaborada de entender la transformación social. El quinto y último momento es la práctica social definida ahora como el punto de la llegada en que los estudiantes alcanzan una comprensión que, según cabe suponer, ya fue encontrado por el profesor en el punto de partida. La práctica social, en este sentido es alterada cualitativamente por la mediación de la acción pedagógica.

Encarado con esa transformación, Saviani, se refiere a la educación como:

"Una actividad que supone una heterogeneidad real y una homogeneidad posible; una desigualdad en el punto de partida y una igualdad en el punto de la llegada".

Saviani considera que la Educación Nueva es extremista al criticar la Educación Tradicional, y en eso hay una inversión del sentido común al definir la Educación Tradicional como inundada de vicios y ninguna virtud. En igual sentido, al analizar las contradicciones evidenciadas en la Escuela Nueva, Saviani trató, por medio de tres tesis, desacreditar el carácter progresivo que esa corriente de pensamiento, ya convertida en sentimiento común, predicaba para la práctica pedagógica. Apuntó con esas tesis refutar la forma de concepción dominante en la educación y justificar una teoria crítica de la educación (no reproductivista); eso le permitió comprender la práctica pedagógica y visualizar los aspectos sobre los cuales una teoria realmente crítica debe centrarse.

Con esas tesis, Saviani trató de mostrar que la tendencia dominante, favorecia a la Escuela Nueva y que era necesario esbozar una teoría critica de la educación, cuya perspectiva pedagógica corresponda a los intereses de la clase trabajadora. La propuesta que abrió los espacios para las fuerzas populares y para que la escuela fuese una institución que posibilite el acceso al conocimiento elaborado, objetivo, históricamente producido y que conduzca a profesores y alumnos a una práctica social que vislumbre el consenso en el punto de la llegada y que fuese capaz de producir transformaciones a favor de una sociedad igualitaria.

Para que la teoría histórico-crítica de la educación sea capaz de ser constituido en una Pedagogía Histórico-crítica debe asumir un posicionamiento acerca de lo que es la educación y qué significa educar a seres humanos. Según Saviani:

"La Pedagogía Crítica implica la claridad de los determinantes sociales de la educación, la comprensión del grado en que las contradicciones de la sociedad marcan la educación y,consecuentemente,el posicionamiento preciso de esas contradicciones y desenmarañar a la educación de las visiones ambigüas, para percibir claramente cuál es la dirección que se imprimirá al problema educativo"

Partiendo de la concepción de la naturaleza humana, propuesta por Marx y Engels de que el hombre necesita producir continuamente durante su existencia y es mediante el trabajo que actúa sobre la naturaleza adaptándola a sus necesidades, Saviani define a la educación como un proceso de trabajo no material (diferente al trabajo material que apunta a la producción de bienes materiales para la subsistencia), en el que el producto no se separa de la producción. El trabajo educativo, dice Saviani, es "el acto de producir directa e intencionalmente, de cada individuo singular, la humanidad que es producida histórica y colectivamente por el conjunto de hombres".

La producción deliberada de la humanidad implica la producción de ideas, valores, de conceptos, hábitos, actitudes, el conocimiento, o sea, la producción del conocimiento o forma por el que el hombre aprehende el mundo y es humanizado. De acuerdo con Saviani, "Lo que no es garantizado por la naturaleza debe ser producido históricamente por los hombres". En ese sentido, el conocimiento objetivo es considerado como la materia prima para la actividad educativa y debe tener la primacía sobre la naturaleza, o sea sobre el saber natural y espontáneo.

Apoyado en Gramsci, Saviani definió la escuela como "una institución cuyo papel consiste en socializar el conocimiento elaborado y no el conocimiento espontáneo, el conocimiento sistematizado y no el conocimiento fragmentado, de la cultura erudita y no de la cultura popular".

El proyecto pedagógico resultante de la Pedagogía Histórico-crítica, se forja en esas reflexiones acerca del concepto de la educación y de la escuela, y la tarea que se propone esa Pedagogía con respecto a la educación de la escuela, según Saviani, implica: 
a. Identificación de las formas más desarrolladas en las que se expresa el conocimiento objetivo producido históricamente, reconociendo las condiciones de su producción y entendiendo sus principales manifestaciones como también las tendencias presentes de transformación:

b. Conversión del saber objetivo en saber escolar de modo que la asimilación por los alumnos no ocupe espacio y tiempos escolares;

c. Proveer de los medios necesarios para que los alumnos asimilen el saber objetivo mientras aprenden el proceso de producción asi como también las tendencias de su transformación.

El gran mérito de Saviani está en no repetir simplemente las ideas de otros grandes maestros, sino que las incorpora a sus reflexiones, a partir de un análisis critico y contextualizado de las circunstancias histórico-culturales en que cada uno de esos autores ha vivido. Y eso le da autonomia y una coherencia con el enfoque que propone para implantar su propuesta pedagógica.

Saviani considera que el método es esencial en el proceso pedagógico, pero él sólo, por si mismo, no es garantizado y ni garantiza una modificación cualitativa de la comprensión de la práctica social. Es necesario que los agentes sociales responsables de la mediación del acto pedagógico, sean agentes sociales activos y verdaderos, desde que ellos son también los elementos objetivos de la práctica social. Es en este sentido que Saviani valoriza y conceptúa a la educación como "una actividad mediadora en el corazón de la práctica social global". En ese punto de vista, Saviani vislumbra en el profesor un agente social, activo y comprometido politicamente con las transformaciones de la sociedad.

\section{RESUMEN DEL DESARROLLO DE LA EXPERIENCIA}

Para verificar los alcances de la PHC y, sobre todo, establecer la conveniencia de su utilización en el ámbito regional, se aplicó un cuestionario de opinión a 42 estudiantes del 4to. y 5to. Años de Secundaria de los 442 alumnos matriculados en tres centros educativos de las provincias de Candarave y Tarata. La muestra ha sido determinada intencionalmente y las unidades muestrales al azar, lo que permite establecer que los datos obtenidos logran un grado de validez confiable. En función al objetivo y la hipótesis planteada, se ha elaborado un cuestionario, con doce preguntas relacionadas a dos aspectos centrales: la metodología de enseñanza empleada por los profesores de cada uno de los centros educacionales estudiados, y la metodología que mejor les parece a los consultados; evidentemente, en esta parte la intencionalidad de las preguntas están dirigidas a demostrar que mediante el uso de la $\mathrm{PHC}$ es posible lograr mejores resultados educativos.

Para lograr un mejor resultado, primero se ha dialogado individualmente con los elementos de la muestra, haciéndoles conocer el propósito del estudio, esclareciendo los aspectos que ellos consideraban necesarios y/o ampliando aquellos que requerian mayores detalles. Una vez cumplida esa primera etapa, se procedió a aplicar los cuestionarios, y en algunas oportunidades se ha tenido que intervenir para ayudar a aclarar aspectos requeridos por ellos.

Los resultados analizados e interpretados nos llevan a determinar las siguientes conclusiones:

1. La educación constituye uno de los elementos fundamentales para el cambic social. En la forma de educar está el resultado o producto; es decir, si se educa para tener un individuo pasivo, dependiente o se educa para tener un individuo activo, renovador o transformador de su sociedad, estará en relación directa el futuro de la sociedad en que uno se desarrolla.

2. Los enfoques o metodologías que los profesores utilizan en las instituciones educativas estudiadas, se caracterizan mayormente por desarrollar pedagogias que hacen al educando un elemento receptivo, pasivo $y$, consecuentemente, poco participativo en las decisiones de su propia colectividad.

3. Los educandos de las instituciones estudiadas, muestran condiciones para el cambio en las metodologias de enseñanza-aprendizaje. Los elementos naturales y fenómenos sociales, económicos, politicos, etc. que operan en su contexto deben constituirse en los fundamentos para la explicación y comprensión de planos superiores de conocimiento y conexión con la realidad local, regional, nacional y mundial.

4. La Pedagogía Histórico -crítica, al comprender métodos y técnicas que posibilitan el engarzamiento entre la realidad y lo deseable a aprender, se erige como una alternativa viable para lograr mejores condiciones para el desarrollo individual y colectivo del futuro habitante de las áreas estudiadas y con posibilidades de lograr mejores condiciones para enfrentar los retos que implica el desarrollo cientifico-tecnológico actual.

\section{BIBLIOGRAFIA}

Freire, P. :Pedagogia del oprimido. Ediciones Tierra Nueva, Montevideo, 1970.

Libaneo, José Carlos.: La Educación y las Tendencias Pedagógicas, Sao Paulo, 1991.

Morales, R., Noé.: La teoría educativa ante el problema de la marginalidad: la formulación de la pedagogía crítica.

Morales, R., Noé.: El hecho educativo como hecho social. En la revista innovaciones educativas, No. 14, Año 2004

Savianl, Dermeval: Escuela y democracia. Editora Cortez Autores Asoc.Sao Pablo, 1984. Saviani, DermevalPedagogia histórico crítica: primeras aproximaciones. Editora Cortez Autores Asociados. Sao Pablo, 1994. 